\title{
SMART COLLEGE BUS TRANSPORT SYSTEM: A REVIEW
}

\author{
Ms. Rahesha Mulla, Ms. Tayyaba Mujawar, Mr. Sumit Sutar, Mr. Rushikesh Shelke, Mr. Shivraj Hippargi \\ Department of Computer Science \& Engineering \\ Sanjay Ghodawat Institute, Kolhapur, Maharashtra, India
}

\begin{abstract}
In almost every college, they provide their bus transportation facility. Under this, updating $\&$ maintaining daily bus records such as bus $\mathrm{km}$, timing, driver and bus details. This every day activity is time consuming \& there is chance of manual mistakes /interference. Paper presented here describes a system that intended to overcome the flaws in the existing system \& bring out accuracy, safety of records. Proposed system uses GPS \& map API for the student, staff to track the bus. Additionally, biometric attendance can be recorded. From the online recorded data on central college server, admin can generate essential report.
\end{abstract}

Keywords- Global Positioning System (GPS), map API, bus tracking, biometric

\section{INTRODUCTION}

In almost every organization, school or college there is their private bus transport service is available. So, they need to keep a record of their vehicle or bus running /driving and the driver of that bus or vehicle. This record contains bus traveling distance (in $\mathrm{km}$ ) and the bus driver's details and they need to keep bus departure, arrival and delay time. They need to keep track of that record in a notebook or register manually. This process is so time-consuming and it needs extra manpower.

There is another problem related to students and staff is that they don't know the real-time location of the bus for which they are waiting for and the time it will take to reach their bus stop. And parents also don't know the location of the bus so, they need to wait. When bus is late then there is a late remark for staff that is traveling through the bus. There is another problem regarding vehicle maintains. To know there are a vehicle needs maintains is so hard.

In this proposed system through GPS we will get the bus running distance, distance will be getting from the map API. We can track the bus from where it is coming and where is its next stop so student, staff, parents do not need to wait they can track the bus in the mobile application. Security head needs to give the daily report of transportation to the concerned authority, this report is generated through this system it will be sent to the concerned authority. After some distance for maintains of the vehicle this system will notify to maintain department that particular bus needs maintenance. And using the biometric devices, we will get the attendance of students and staff on time so that if the bus was late then the staff cannot get late remark and if a student is not in the bus after a certain stop parent will get notified that their child was not in the bus .Information about the bus acquired by integrating the GPS device and biometric device in the bus.

This will help to reduce man power and time for this work. There are many systems are available to bus tracking and monitoring student in bus. But this system will track the bus, monitor student and generate report using GPS and map API.

This system will be easy to access because there is no any manual work needs to do, making system smart which works automatically.

This system has first component is GPS, GPS sends coordinates and we will locate bus on map using map API that will track on mobile or desktop. There is only user need to enter vehicle number and user will get the real time location of the vehicle. This is about the tracking of vehicle. Using map data, we get travelling distance of vehicle and we will get the bus current distance traveled status. From this data we will provide maintenance alert for maintain vehicle.

This system also monitors student and staff who are traveling through the bus. We fix fingerprint scanner for monitoring. This report will send to the central system.

From this all collected information we are generating report for higher authority so they will check transport status. If there is any mistake or error, they will take action. It also improves data security no one will be change data because everything is system generated data is provided.

\section{EXISTING WORK}

Existing android-based school bus tracking and student monitoring system, which helps parents keeps track of their ward's bus facility provided by college. This application is handled by four categories of 
users: parents, faculty/teacher/staff, bus drivers, and admin of the system. Admin handles all the users and all emergency situations about main events that are happening or that would happen in a particular day. Faculty is in charge of all children in a bus and makes sure that they have boarded the bus or not. Driver is in charge of notifying the start and end of journey. Only after his/her confirmation shall the bus's location starts transmitting to parents. Parents can watch the live location of the bus and ensure that their child have boarded or not by just reviewing. Each child is identified by his/her own unique ID. This system is using android application, this existing work implements bus tracking and student attendance monitoring using an android application, GPS, GPRS, google map API, firebase, cloud server [1]. This system has an android application for bus tracking and student monitoring it needs to use manually so it here is security and accuracy is not maintained.

Application-based bus tracking system, preprocessing technique and Kalman filter is a technique which is implemented for increasing the accuracy of the location. This system just improves the accuracy of tracking. Existing system have time lags so they are fixing this problem using preprocessing and Kalman filter [2].

GPS based bus tracking system, system track the bus and get the arrival time of the bus on the bases of speed with which the bus is moving and the average velocity from the historical trends under same day and time of day conditions. This proposed system is divided into two subsystems. First, GPS based system that tracks the current location of the bus and the passenger to calculate the distance between the two. Also tracks the real time speed of the bus. Secondly the prediction system, which calculates the average velocity of each segment from the data that captures the historical trends of traffic on the basis of different attributes like segment, day, time, volume of traffic and crossings in the segment. The proposed system is based on the client server technology, which consists of two types of client-side application and the server side.[3]

An accurate real time GPS tracking system use GPS and GSM service for real time location of object. This system consists of GPS receiver, GSM modem and microcontroller. Mobile tracked device receives its coordinates form GPS and send it tracking center through SMS via GSM modem. [4]

\section{PROPOSED ARCHITECTURE}

In the currently available smart bus/transportation system, it provides basic services such as bus tracking and attendance system using RF-ID and manual entry of bus reading, bus driver name, bus inout time, bus number. The proposed system contains bus tracking, attendance system using biometric device and automatic entry of bus arrival and departure timing. The system also provides the running distance of the bus that will be added to the existing database and generates total traveling distance of the bus. Using this all information we will generate a report that will be provided to transportation incharge. Because of this system, we would need less time and fewer men power for this work this is the main advantage of the proposed system.

The proposed system uses the GPS that will be located in the vehicle, GPS will send data to as input to the system. Data is nothing but longitude and latitude. Using longitude and latitude we will track the bus on the map using map API. Using the longitude and latitude we can get the start and end point of the trip and we are going to get the distance from the map API. From map we will get the traveled distance of bus and we are going to store that to the data base for that current date trip detail and adding to existing traveled distance so we will gate the reading of the bus and form this we going to generate report. From the generated data we are also providing maintenance notification.

System also generates attendance report of student and staff that will be provided to the authorized person for system monitoring.

System also provides live vehicle geolocation tracking for users. User can track the vehicle by its number. After entering number bus will be located on map. User has live tracking access on mobile or desktop they can track vehicle in browser just entering vehicle number.

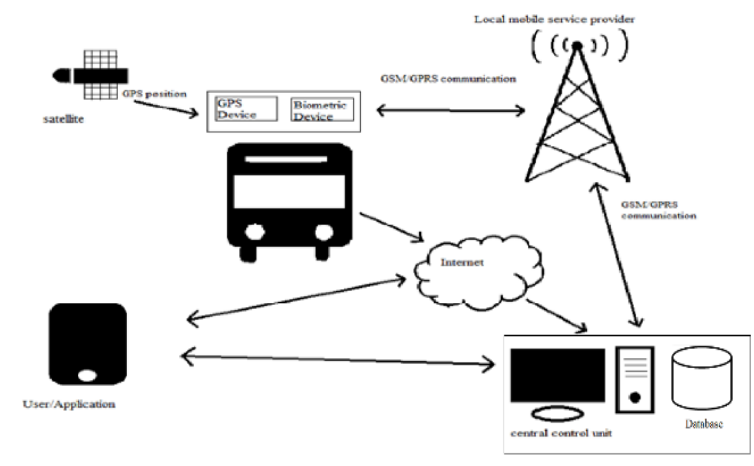

Fig. 1 System architecture

A. GPS: The GPS is a U.S.-owned utility that provides users with positioning, navigation, and timing (PNT) services. This system consists of three segments: the space segment, the control segment, 
and the user segment. The U.S. Air force develops, maintains, and operates the space and control segments.

The basic GPS system consists of two segments having the purpose of providing a consistent and reliable tracking and timing service to all the GPS users all over the world. These segments include space segment \& control segment.[5] GPS receives vehicle geolocation coordinates from satellite. It uses travel time or time of flight (TOF) measurements in determining its position. GPS TOFSi, Rx measurement is the time-lapse of a signal to reach at the receiver, $\mathrm{Rx}$, from $\mathrm{i}^{\text {th }}$ satellite, $\mathrm{Si}$. For acceptable level of accuracy in position estimate GPS requires minimum of four TOF measurements from individual satellites. [8]

Hence, Extended Kalman Filter Estimator (EKFE) or KFE with linearized measurement equations is used to estimate the unknown receiver position. The taylors series first order approximated linear form 10 of Eq.1, computed at $x \mathrm{R}^{\wedge}$ is given in Eq. which is used in framing the observation matrix, $\Omega$ in KFE.[8]

$$
T O F_{S i, R x}=f(S, R x)=\sqrt{\left(X_{S i}-X_{R x}\right)^{2}+\left(Y_{S i}-Y_{R x}\right)^{2}+\left(Z_{S i}-Z_{R x}\right)^{2}}
$$

$$
f(S, R x) \cong f\left(S, R^{\wedge} x\right)+f^{\prime}\left(S, R^{x}\right)\left(R x-R^{\wedge} x\right)
$$

Here, TOFSi,Rx is the time of flight between $i^{\text {th }}$ satellite, $\mathrm{Si}$ and receiver, $\mathrm{Rx}, \mathbf{S}=$ (xsi,ysi,zsi) is the three dimensional position coordinates of $i^{\text {th }}$ satellite, $\mathbf{R x}=(\mathrm{xRx}, \mathrm{yRx}, \mathrm{zRx})$ is the three dimensional position coordinates of receiver and f'represents the first order derivative of function $f(S, R x)$. The Eq. can also be represented as,[8]

$$
\begin{aligned}
& \mathrm{f}(\mathrm{S}, \mathrm{Rx})-\mathrm{f}\left(\mathrm{S}, \mathrm{R}^{\wedge} \mathrm{x}\right) \cong \mathrm{f}^{\prime}\left(\mathrm{S}, \mathrm{R}^{\wedge} \mathrm{x}\right)\left(\mathrm{Rx}-\mathrm{R}^{\wedge} \mathrm{x}\right) \Rightarrow \delta T O F_{S i, R x} \\
&\left.\cong \frac{\partial f(S, R x)}{\partial R x}\right|_{R x=R^{x}} \delta R x \Rightarrow \delta T O F_{S i, R x} \cong \Omega\left(\mathrm{S}, \mathrm{R}^{\wedge} \mathrm{x}\right) \delta \mathrm{Rx}
\end{aligned}
$$

B. GSM/GPRS Communication: GSM (Global System for Mobile communication) is a digital mobile network that is widely used by mobile phone users in Europe and other parts of the world. GSM uses a variation of time division multiple access (TDMA) and is the most widely used of the three digital wireless telephony technologies: TDMA, GSM and Code-Division Multiple Access (CDMA). GSM digitizes and compresses data, then sends it down a channel with two other streams of user data, each in its own time slot. It operates at either the 900 megahertz $(\mathrm{MHz})$ or 1,800 $\mathrm{MHz}$ frequency band. Where we are using this for establishing internet connection. Here we are using internet connection for sending collected GPS and biometric device data to central control unit.
C. Central Control Unit: Central control unit receive data from GPS and biometric device through internet. When data will be received the data will be stored to data base and other data operation will be performed here. In central control unit there is report generation and other operations are performed.

D. User and Application: At the end user will access needed data as per his/her permissions. Data access will be including bus tracking for student, staff and admin. Admin will have all access like daily transport details and monitoring of travelers. A mobile application, most commonly referred to as an app, is a type of application software designed to run on a mobile device, such as a smartphone or tablet computer. Mobile applications frequently serve to provide users with similar services to those accessed on PCs. Apps are generally small, individual software units with limited function. This use of app software was originally popularized by Apple Inc. and its App Store, which offers thousands of applications for the iPhone, iPad and iPod Touch.

The architecture components have following function,

A. Receive data from GPS: The systems main goal is to receive data from the GPS which is located in the bus. Data consists of co-ordinates of bus and it will be stored database on a central sever.

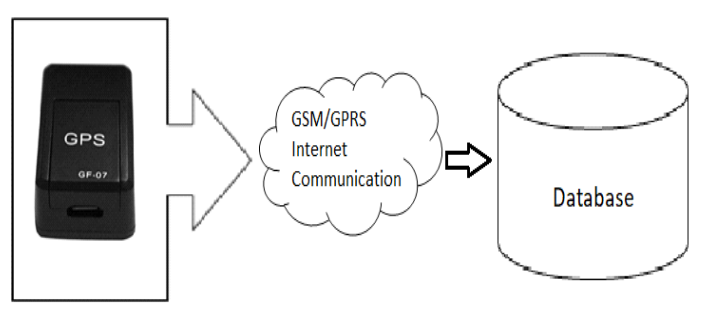

Fig. 2 Data receiving and storing in database

In this module GPS sends coordinates to system through the internet that will show live location of the vehicle and these coordinates are also stored in data base for data processing. We will get internet access using GSM module. A PHP code has been written to connect to MYSQL database, extract and process the longitude and latitude. We will code GSM for this function using the UNO board. 
B. Data analysis and Data processing: Using received data we can track/ find location of bus and we can get travelled distance of bus.

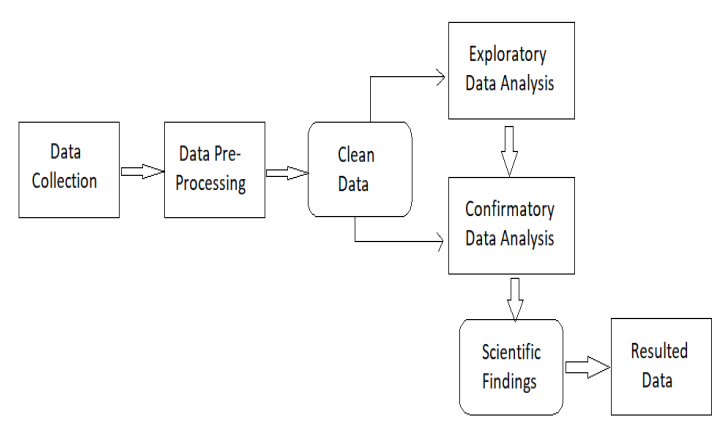

Fig. 3 Data analysis and data processing

Here we are going to generate useful data. Data contain departure, arrival and delay time. It generates traveling distance report using map API. This all data should be sent to transport incharge. So, he/she can do needful analysis of generated report.

C. Finger print recognition: this module will record biometric fingerprint-based attendance of student and staff. This data will be sent to central control unit. So, it will generate notification of attendance. The basic fingerprint recognition system consists of training and testing phase. Training phase consist of model building and testing phase performs recognition. Basically, system can be described as with four stages: firstly, the sensor which is used for enrollment $\&$ recognition to capture the biometric data. Secondly, the pre-processing stage which is used to remove unwanted data and increase the clarity of ridge structure by using enhancement technique. Thirdly, feature extraction stage which takes the input from the output of the pre-processing stage to extract the fingerprint features. Fourthly, the matching stage is to compare the acquired feature with the template in the database. Finally, the database which stores the features for the matching stags.[6] Biometric device matches stored biometric fingerprint and collect traveler and bus driver information.

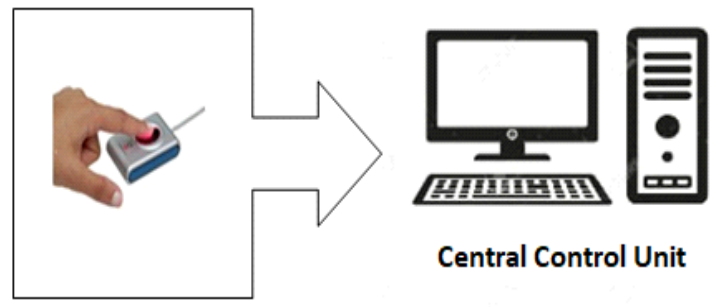

Fig. 4 Finger print recognition

Module gathers attendance of every student using biometric fingerprint scanner and it will be sent to the system and store it in the data base using minutiae correspondence algorithm

Algorithm: Minutiae correspondence algorithm

1: Input: Latent minutiae template with nl minutiae and reference print minutiae template with $\mathrm{nr}$ minutiae

2: Output: Minutiae correspondences

3: Compute the $\mathrm{nl} \times \mathrm{nr}$ minutiae similarity matrix using Eq.

4: Select the top $\mathrm{N}$ minutiae correspondences based on the above minutiae similarity matrix

5: Construct $\mathrm{H} 2$ based on these $\mathrm{N}$ minutiae pairs

6: Remove false minutiae correspondences using Algorithms 2 and 4

7: Construct $\mathrm{H} 3$ for the remaining minutiae pairs

8: Remove false minutiae correspondences using Algorithms 3 and 4

9: Output final minutiae correspondences. [7]

D. Report generation: Now these data will contain bus and bus member's information. Information such as distance travelled by bus, location of bus, departure, arrival and delay time, biometric attendance. This information will be sent to concerned authority as transportation system report.

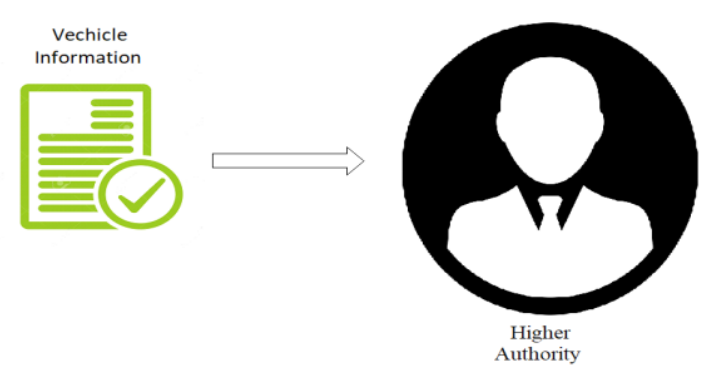

Fig. 5. Report generation

Here we will get the all information of vehicle that will be send to the higher authority. 


\section{CONCLUSION}

This proposed system is used for real time transportation applications with major modification to existing transportation system to avoid the manual work and make it as fully automated, accurate on software basis. It uses basic measurements of distance in $\mathrm{km}$ between two locations and provides necessary details of each and every route for students and staffs to easily pick up buses or any other conveyance possible on the specified route. Specific location details are provided to the user along with biometric service. Student and staff can identify the bus correctly. Extension to the proposed system will cover real time nearby emergency points, hospitals and maintenance garages.

\section{ACKNOWLEDGEMENT}

The presented paper is an outcome of motivation received from "An Android Application for School Bus Tracking and Student Monitoring System" by Jisha R C, Mathews P Mathews, Sidharth P Kini, Vineeth Kumar, Harisankar U V, Shilpa M. We tried to partially implement Smart College Bus Transport System as a part of UG student projects to learn Fog Computing and to work with new technologies.

\section{REFERENCES}

[1] Jisha, R. C., Mathews, M. P., Kini, S. P., Kumar, V., Harisankar, U. V., \& Shilpa, M. (2018). An Android Application for School Bus Tracking and Student Monitoring System. 2018 IEEE International Conference on Computational Intelligence and ComputingResearch

(ICCIC). doi:10.1109/iccic.2018.8782320.

[2] Jain, S., Trivedi, A., \& Sharma, S. (2019). Application Based Bus Tracking System. 2019 International Conference on Machine Learning, Big Data, Cloud and Parallel Computing (COMITCon). doi:10.1109/comitcon.2019.8862254

[3] Singla, L., \& Bhatia, P. (2015). GPS based bus tracking system. 2015 International Conference on Computer, Communication and Control (IC4). doi:10.1109/ic4.2015.7375712

[4] Abdallah Dafallah, H. A. (2014). Design and implementation of an accurate real time GPS tracking system. The Third International Conference on eTechnologies and Networks for Development (ICeND2014). doi:10.1109/icend.2014.6991376
[5] Mulla, A., Baviskar, J., Baviskar, A., \& Bhovad, A. (2015). GPS assisted Standard Positioning Service for navigation and tracking: Review \& implementation. 2015 International Conference on Pervasive

(ICPC). doi:10.1109/pervasive.2015.7087165 Computing

[6] Ali, M. M. H., Mahale, V. H., Yannawar, P., \& Gaikwad, A. T. (2016). Overview of fingerprint recognition system. 2016 International Conference on Electrical, Electronics, and Optimization Techniques (ICEEOT). doi:10.1109/iceeot.2016.7754900

[7] Cao, K., \& Jain, A. K. (2018). Automated Latent Fingerprint Recognition. IEEE Transactions on Pattern Analysis and Machine Intelligence, 11. doi:10.1109/tpami.2018.2818162

[8] Laveti, G., Rao, G. S., \& Bidikar, B. (2016). Modified Kalman Filter for GPS Position Estimation over the Indian Sub Continent. Procedia Computer Science, 87, 198203. doi:10.1016/j.procs.2016.05.148 